\title{
Influence of quasibound states on the carrier capture in quantum dots
}

\author{
Magnúsdóttir, Ingibjörg; Uskov, A.V.; Ferreira, R.; Bastard, G.; Mørk, Jesper; Tromborg, Bjarne
}

Published in:

Applied Physics Letters

Link to article, DOI:

$10.1063 / 1.1526911$

Publication date:

2002

Document Version

Publisher's PDF, also known as Version of record

Link back to DTU Orbit

Citation (APA):

Magnúsdóttir, I., Uskov, A. V., Ferreira, R., Bastard, G., Mørk, J., \& Tromborg, B. (2002). Influence of quasibound states on the carrier capture in quantum dots. Applied Physics Letters, 81(23), 4318-4320. https://doi.org/10.1063/1.1526911

\section{General rights}

Copyright and moral rights for the publications made accessible in the public portal are retained by the authors and/or other copyright owners and it is a condition of accessing publications that users recognise and abide by the legal requirements associated with these rights.

- Users may download and print one copy of any publication from the public portal for the purpose of private study or research.

- You may not further distribute the material or use it for any profit-making activity or commercial gain

- You may freely distribute the URL identifying the publication in the public portal 


\title{
Influence of quasibound states on the carrier capture in quantum dots
}

\author{
I. Magnusdottir ${ }^{\text {a) }}$ \\ COM, Technical University of Denmark, Building 345w, DK-2800 Lyngby, Denmark
}

A. V. Uskov

Lebedev Physical Institute, Leninsky prospect 53, 117924 Moscow, Russia

R. Ferreira and G. Bastard

Laboratoire de Physique de la Matiere Condensée, ENS, 24 rue Lhomond, F-75005 Paris, France

J. Mørk and B. Tromborg

COM, Technical University of Denmark, Building 345w, DK-2800 Lyngby, Denmark

(Received 2 July 2002; accepted 9 October 2002)

The interaction of carriers in quantum-dot quasibound states with longitudinal optical phonons is investigated. For a level separation between the quasibound state and a discrete quantum-dot state in the vicinity of the phonon energy, a strong electron-phonon coupling occurs. A mixed electronphonon mode-polaron - is formed. The finite lifetime of the phonons is shown to give rise to another type of carrier capture into quantum dots. (c) 2002 American Institute of Physics. [DOI: $10.1063 / 1.1526911]$

An important characteristic of self-assembled quantumdot (QD) materials is the timescale on which carriers are captured into the dots and relax to their ground state. These processes are mediated by carrier-phonon and carriercarrier interaction (Auger processes). ${ }^{1,2}$ In calculations of carrier capture rates in QDs by longitudinal optical (LO) phonon emission ${ }^{1}$ and Auger scattering, ${ }^{2,3}$ the continuum wave functions have been considered to be plane waves and hence, the influence of waves scattered by the QD, i.e., quasibound states, has been neglected. At certain QD sizes and wavelengths of an incident carrier, the QD acts as a Fabry Perot mirror, in which the carrier "bounces" back and forth, giving rise to a long lived state of finite linewidth that resembles the discrete QD states. In systems of higher dimensionality, carrier relaxation via emission of LO phonons is efficient. In the zero-dimensional QD systems, LO phonon relaxation between the discrete QD states is often considered impossible unless the energy level separation equals the LO phonon energy, leading to a so-called phonon bottleneck. ${ }^{4}$ Recently, it has however been shown both theoretically and experimentally ${ }^{5,6}$ that due to the discretization of the electronic spectrum, carriers in bound QD states couple strongly to LO phonons, and form polarons. This implies that the coupling of bound electrons to LO phonons cannot be treated in the frame of Fermi's golden rule and that the energy relaxation in QDs does not correspond to irreversible onephonon processes. Instead, the finite phonon lifetime, measured in bulk semiconductors to be about $10 \mathrm{ps}$ at low temperatures ( $2 \mathrm{ps}$ at room temperature) ${ }^{7}$ leads to a relaxation of the polaron. An analogue of such a coupled electron-LO phonon system is a coupled exciton-photon system in a microcavity in which energy is periodically exchanged between exciton and photon modes. ${ }^{8}$ This is known as Rabi oscillation.

The question arises to which extent the standard pertur- bative approach for calculating carrier capture rates into QDs via LO phonon emission is valid, when taking into account the QD quasibound states. In this letter, we investigate the carrier-LO phonon coupling for carriers in quasibound QD states and we shall demonstrate that they couple strongly with LO phonons. Quasibound states have been investigated in quantum wells ${ }^{9,10}$ and were shown to induce strong oscillations in the carrier capture rate versus well thickness. ${ }^{11}$

We use the model of a spherical QD of radius $a$ with finite confinement potential, $V_{0}$, in the effective-mass approximation where the states are characterized by the angular momentum quantum numbers $(\ell, m)$. We assume for simplicity that one discrete state is bound to the dot (with $\ell$ $=0$ ), hereafter labeled $|S\rangle$, and the continuous part of the energy spectrum is characterized by the wave number, $k_{\nu}$, and the angular momentum quantum numbers $\left(\ell_{\nu}, m_{\nu}\right)$. In this simple model for QDs, we have the advantage of knowing the electronic wave functions for all energies. For the complex shape and potential of actual self-assembled QDs, the wave functions for bound states are not very well determined and little or nothing is known about wave functions of quasibound states.

We only include one quasibound state of definite angular momentum $\left(l_{\nu}=1\right)$, labeled $|\nu\rangle$. This state will become bound as the dot becomes larger. The electronic continuum is discretized by enclosing the QD in a large sphere of radius $R_{b}$. The position in energy of a quasibound state can be traced by a local increase in the probability that the carrier is present within the QD volume, shown in Fig. 1(a) for an electron with $l_{\nu}=1 . E=0$ is set at the bottom of the QD. $V_{0}$ and $a$ are chosen such that a narrow resonance appears at $E_{1}=76.58 \mathrm{meV}$, one LO phonon energy, $\hbar \omega_{\mathrm{LO}}(=35 \mathrm{meV})$, above the discrete QD state.

We now turn to the carrier-LO phonon interaction. The Hamiltonian for the electron-phonon system is

$$
H=H_{e}+H_{\mathrm{ph}}+H_{e-\mathrm{ph}},
$$

${ }^{a)}$ Electronic mail: im@com.dtu.dk 


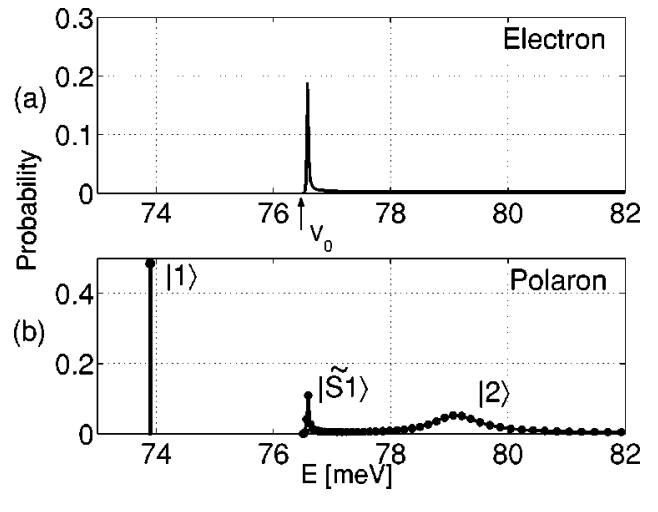

FIG. 1. (a) The probability that an electron state with $\ell_{\nu}=1$ in the continuous part of the energy spectrum is present within the volume of the QD, neglecting the carrier-LO phonon interaction. A quasibound state is situated at $E_{1}=76.58 \mathrm{meV}$ with a FWHM $\hbar \Gamma_{e}=0.025 \mathrm{meV}$. The discrete $\ell=0$ state (not shown) is situated at $E=41.59 \mathrm{meV}, \hbar \omega_{\mathrm{LO}}$ below the quasibound state. (b) Taking carrier-LO phonon interaction into account, two polaron states $(|1\rangle,|2\rangle)$ appear. One, $|1\rangle$, is discrete and at $E_{0}=73.9 \mathrm{meV}$. $|2\rangle$ is a broader level centered at $E=79.1 \mathrm{meV}$ and the Rabi splitting is $E_{\mathrm{Rabi}}=5.2 \mathrm{meV}$. We use $a=8.55 \mathrm{~nm}$, the carrier mass $m^{*}=0.067 m_{0}, V_{0}=76.49 \mathrm{meV}$, and $R_{b}=1500 \mathrm{~nm}$.

where $H_{e}$ is the electronic and $H_{\mathrm{ph}}$ the phonon Hamiltonian. The electron-phonon interaction is described by the Fröhlich Hamiltonian

$$
H_{e-\mathrm{ph}}=\sum_{\mathbf{q}}\left(\alpha^{*}(\mathbf{q}) e^{-i \mathbf{q} \cdot \mathbf{r}} \hat{a}_{\mathbf{q}}^{\dagger}+\alpha(\mathbf{q}) e^{i \mathbf{q} \cdot \mathbf{r}} \hat{a}_{\mathbf{q}}\right),
$$

where $\hat{a}_{\mathbf{q}}\left(\hat{a}_{\mathbf{q}}^{\dagger}\right)$ is a destruction (creation) operator for an optical phonon mode of wave vector $\mathbf{q}$ and

$$
\alpha(\mathbf{q})=-i \sqrt{\frac{\hbar \omega_{\mathrm{LO}} e^{2}}{2 \epsilon_{0} V q}\left(\frac{1}{\epsilon_{\infty}}-\frac{1}{\epsilon_{r}}\right)},
$$

where $\epsilon_{\infty}\left(\epsilon_{r}\right)$ is the high-frequency (static) dielectric constant, $\epsilon_{0}$ is the permittivity constant and $V$ is the quantization volume. The phonon continuum is discretized and we restrict ourselves to polaron states with the zero-phonon mode or modes for which the number of phonons is at most one. The polaron wave function can be expressed as

$$
|\Psi\rangle=\sum_{\nu} a_{\nu}\left|\nu, 0_{\mathrm{ph}}\right\rangle+\sum_{i} \gamma_{i}\left|S, 1_{\mathbf{q} i}\right\rangle .
$$

The diagonalization of the total Hamiltonian couples a few of the phonon modes to the electron states leaving a large amount of the phonon bath "intact". The coupled electronphonon states form the polaron states. Figure 1(b) shows the probability of presence within the QD volume for the polarons. Pure phonon modes have been excluded and only modes with nonzero projection onto the states $\left|\nu, 0_{\mathrm{ph}}\right\rangle$ are taken into account. We find that two polaron states are formed, labeled $|1\rangle$ and $|2\rangle$ as in Ref. 12. The Rabi splitting between $|1\rangle$ and $|2\rangle$ is given by $E_{\mathrm{Rabi}}=5.2 \mathrm{meV}$, giving a measure of the Fröhlich coupling strength. The lifetime of both the constituents of the polaron, i.e., electrons and phonons, must be much larger than half the Rabi oscillation period. Otherwise, the Rabi oscillation is destroyed, and there is no chance for the polaron to be formed. Equivalently,

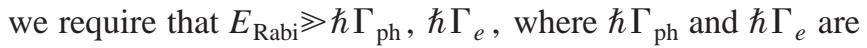
the phonon and electron linewidths, respectively. The electron linewidth is $\hbar \Gamma_{e}=0.025 \mathrm{meV}$, and the phonon linewidth Downloaded 12 Mar 2010 to 192.38.67.112. Redistribution subject

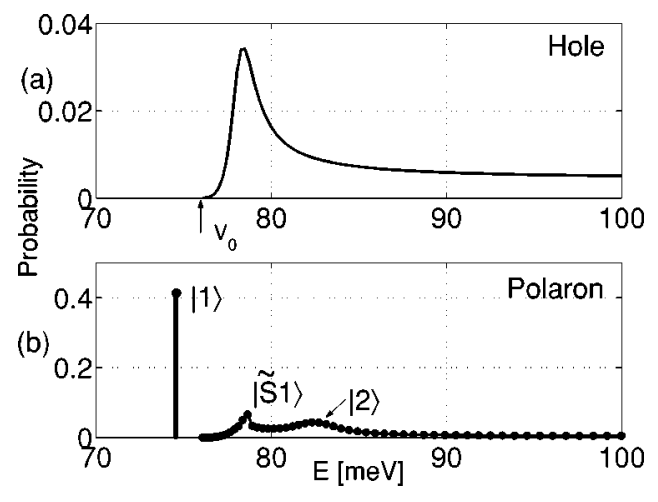

FIG. 2. Same as in as Fig. 1 except for holes. The quasibound state seen in (a) when carrier-LO phonon interaction is neglected is broad compared to the case for electrons. It is situated at $E_{1}=78.34 \mathrm{meV}$ with $\hbar \Gamma_{h}=2.1 \mathrm{meV}$. The discrete $\ell=0$ state (not shown) is situated at $E_{0}=43.5 \mathrm{meV}, \hbar \omega_{\mathrm{LO}}$ below the quasibound state. The polaron states appearing when the interaction is taken into account (b) contain a discrete level, $|1\rangle$, at $E=74.6 \mathrm{meV}$. The state $|2\rangle$ is centered at $E=82.3 \mathrm{meV}$, so the Rabi splitting is $E_{\text {Rabi }}=7.7$ $\mathrm{meV}$. The QD radius is $a=3.662 \mathrm{~nm}$, the carrier mass $m^{*}=0.34 m_{0}$, $V_{0}=76.0 \mathrm{meV}$, and $R_{b}=400 \mathrm{~nm}$.

is not larger than about $0.3 \mathrm{meV}$ (taking the roomtemperature phonon dephasing rate, i.e., $\Gamma_{\mathrm{ph}}=0.5 \mathrm{ps}^{-1}$ ), so the condition is fulfilled here. As an important result, we find that $|1\rangle$ corresponds to a discrete state confined to the QD with a probability close to $50 \%$, while the probability for the electronic state $|S\rangle$ is about $80 \%$.

A reminescence of the quasibound state is seen between $|1\rangle$ and $|2\rangle$, labeled $|\tilde{S} 1\rangle$, recognized by a finite linewidth and the same shape as the electronic quasibound state. The ground state $\left|S, 0_{\mathrm{ph}}\right\rangle$ has not been taken into account in the calculation, since the Fröhlich interaction merely results in a small shift of the S level. ${ }^{12}$ The results in Fig. 1 can be shown to be independent of the azimuthal quantum number, $m_{\nu}$, so the polaron states are triply degenerate due to the $\left(2 \ell_{\nu}+1\right)$-degeneracy of the carrier quasibound state.

Figure 2(a) shows the probability for a hole quasibound state that is about a 100 times broader than the electron resonance. Figure 2(b) shows the probability when the Fröhlich interaction has been taken into account. Here, polarons are also formed. We obtain a Rabi splitting $E_{\text {Rabi }}=7.7 \mathrm{meV}$ which fulfills again $E_{\text {Rabi }} \gg \hbar \Gamma_{h}, \hbar \Gamma_{\mathrm{ph}}$. It should be emphasized here that within the strong coupling regime, it is no longer possible to use the notion of phonon absorption/ emission. The relaxation of a polaron as a whole can nevertheless take place, due to an anharmonic decay of its phonon part. It is known in bulk structures that LO phonons disintegrate into two less energetic phonons. ${ }^{7}$ The decay frequency has been measured in bulk structures to be $\Gamma_{\mathrm{ph}}=0.1 \mathrm{ps}^{-1}$ at low temperatures and $0.5 \mathrm{ps}^{-1}$ at room temperature. The phonon decay has not been measured in QDs but the lifetime of confined LO phonons in QDs has been calculated to be of the same order. ${ }^{13}$ In a relaxation process from $|1\rangle$ to $|S 0\rangle$, we have $E_{|1\rangle}-E_{|S 0\rangle}=E_{\mathrm{LO}}+E_{\mathrm{TA}}$ if the relaxation arises from disintegration into an LO and a transverse acoustic phonon. Other relaxation paths are also possible, see Ref. 7. This relaxation process does not require the electronic levels to be separated by one LO phonon, since it concerns relaxation of the polaron as a whole, due to phonon-phonon interaction on polaron states. For instance, the relaxation rate of a $|1\rangle$ state can be calculated by weighing $\Gamma_{\text {ph }}$ by the phonon part
AlP license or copyright; see http://apl.aip.org/apl/copyright.jsp 

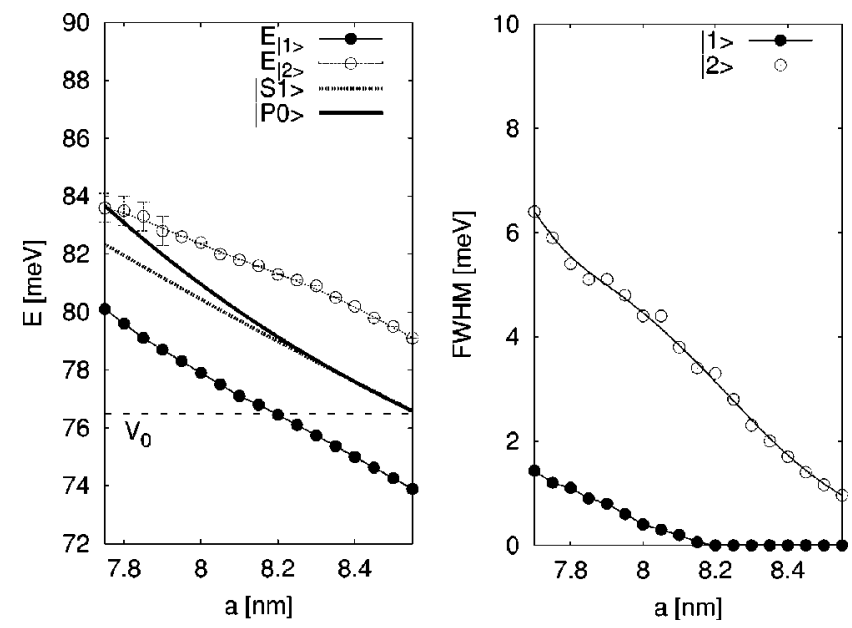

FIG. 3. The left-hand side panel shows the energy position of the polaron states $|1\rangle$ and $|2\rangle$ as a function of QD radius along with the zero-phonon quasibound level peak $(|P 0\rangle)$ and the one-phonon sideband of the $\mathrm{S}$ level $(|S 1\rangle)$. The horizontal line shows the onset of the continuum (i.e., the barrier position). The parameters are the same as in Fig. 1. The polaron levels $|1\rangle$ and $|2\rangle$ are observable down to $a=7.75 \mathrm{~nm}$. The quasibound state broadens as its energy increases (the dot size decreases) and hence, the polaron levels also broaden. The linewidth (FWHM) of levels $|1\rangle$ and $|2\rangle$ is shown in the right-hand side panel for the same radius interval.

of the total probability $\langle\Psi \mid \Psi\rangle$. This yields relaxation times for both electron and hole polarons of about 4 ps at $T=300$ $\mathrm{K}$ ( $20 \mathrm{ps}$ at low temperatures). We note here that in quantum wells, the average capture time (without the polaron effect) has been calculated to be around $100 \mathrm{ps} .{ }^{11}$ Figure 3 shows the radius dependence of the polaron levels for the same parameters as in Fig. 1. The polaron levels $|1\rangle$ and $|2\rangle$ can be seen down to $a=7.75 \mathrm{~nm}$. The quasibound state broadens as its energy increases (the dot size decreases), since at higher energies, the incident carrier "feels" the presence of the confinement potential less. Hence, the polaron levels broaden as well. For smaller radii than the minimal radius shown here, the width of the levels becomes too large. This is seen in the right-hand side panel of Fig. 3, where the linewidth [full width at half maximum (FWHM)] is given for the same radius interval. At $a=8.6 \mathrm{~nm}$, the quasibound level becomes bound. At $a=8.2 \mathrm{~nm}$, the polaron level $|1\rangle$ becomes bound, as depicted by the zero linewidth in the right-hand side panel beyond $8.2 \mathrm{~nm}$.
In the preceding calculations, we have neglected the influence of carrier-carrier interaction on linewidths of quasibound states. For instance, under optical excitation, a large amount of carriers exist in the vicinity of the dots and charge fluctuations might increase the linewidth. Furthermore, effects of thermal broadening have not been taken into account. Dephasing times of excited states have been measured in self-assembled QDs in a four-wave mixing experiment to be around $60 \mathrm{ps}$ at $5 \mathrm{~K}$, compared to a ground-state dephasing time of 372 ps. ${ }^{14}$ To our knowledge, nothing has been reported so far on dephasing times of quasibound states.

In summary we have demonstrated that carriers in quasibound QD states couple strongly with LO phonons. We have shown that the finite LO-phonon lifetime leads to a relaxation of the polaron to the discrete QD ground state. In the weak-coupling regime, i.e., when the energy level separation differs significantly from $\hbar \omega_{\mathrm{LO}}$, carrier capture rate can be calculated with the conventional perturbative approach.

The work of one of the authors (A.V.U.) was supported by the Russian Federal Program Integration (Project No. A0155), RFBR (Project No. 01-02-17330), INTAS (Project No. 2001-0571), the Danish Research Council within the framework of program SCOOP, and the Otto Moensted Foundation.

${ }^{1}$ R. Ferreira and G. Bastard, Appl. Phys. Lett. 74, 2818 (1999).

${ }^{2}$ A. V. Uskov, J. McInerney, F. Adler, and H. Schweizer, Appl. Phys. Lett. 72, 58 (1998).

${ }^{3}$ A. V. Uskov, Y. Boucher, J. Le Bihan, and J. McInerney, Appl. Phys. Lett. 73, 1499 (1998).

${ }^{4}$ H. Benisty, C. M. Sotomayor-Torres, and C. Weisbuch, Phys. Rev. B 44, 10945 (1991).

${ }^{5}$ T. Inoshita and H. Sakaki, Phys. Rev. B 56, R4355 (1997).

${ }^{6}$ S. Hameau, Y. Guldner, O. Verzelen, R. Ferreira, G. Bastard, J. Zeman, A. Lemaitre, and J. M. Gérard, Phys. Rev. Lett. 83, 4152 (1999).

${ }^{7}$ F. Vallée and F. Bogani, Phys. Rev. B 43, 12049 (1991).

${ }^{8}$ C. Weisbuch, M. Nishioka, A. Ishikawa, and Y. Arakawa, Phys. Rev. Lett. 69, 3314 (1992).

${ }^{9}$ H. Luo, N. Dai, F. C. Zhang, N. Samarth, M. Dobrowolska, J. K. Furdyna, C. Parks, and A. K. Ramdas, Phys. Rev. Lett. 70, 1307 (1993).

${ }^{10}$ G. Bastard, Phys. Rev. B 30, 3547 (1984).

${ }^{11}$ J. A. Brum and G. Bastard, Phys. Rev. B 33, 1420 (1986).

${ }^{12}$ O. Verzelen, R. Ferreira, and G. Bastard, Phys. Rev. B 62, R4809 (2000).

${ }^{13}$ X. Q. Li and Y. Arakawa, Phys. Rev. B 57, 12285 (1998).

${ }^{14}$ D. Birkedal, K. Leosson, and J. M. Hvam, Phys. Rev. Lett. 87, 227401 (2001) 